\title{
DOUBLE OPTIMAL STOPPING IN THE FISHING PROBLEM
}

\author{
ANNA KARPOWICZ, ${ }^{*}$ Wroctaw University of Technology
}

\begin{abstract}
In this paper we consider the following problem. An angler buys a fishing ticket that allows him/her to fish for a fixed time. There are two locations to fish at the lake. The fish are caught according to a renewal process, which is different for each fishing location. The angler's success is defined as the difference between the utility function, which is dependent on the size of the fish caught, and the time-dependent cost function. These functions are different for each fishing location. The goal of the angler is to find two optimal stopping times that maximize his/her success: when to change fishing location and when to stop fishing. Dynamic programming methods are used to find these two optimal stopping times and to specify the expected success of the angler at these times.
\end{abstract}

Keywords: Fishing problem; optimal stopping; dynamic programming; semi-Markov process; infinitesimal generator

2000 Mathematics Subject Classification: Primary 60G40

Secondary $60 \mathrm{~K} 15$

\section{Introduction}

The solution to the double optimal stopping problem, in the so-called 'fishing problem', will be presented. Starr [10] was the first to consider a basic version of this problem, with further generalizations given later by Starr and Woodroofe [11], Starr et al. [12], and Kramer and Starr [8]. For a detailed review of the literature on the fishing problem, see [5, p. 1]. The simple formulation of our double optimal stopping problem is as follows. An angler buys a fishing ticket that allows him/her to fish for a fixed time $t_{0}$. There are two locations to fish at the lake and the angler can change his/her location at any time $s$. The fish are caught according to a renewal process $\left\{N_{i}(t), t \geq 0\right\}$, where $N_{i}(t)$ denotes the number of fish caught during time $t$ at location $i=1,2$. Let $T_{i, n}$ denote the capture time of the $n$th fish at location $i$ (we fix $T_{1,0}=0$ and $T_{2,0}=s$ ). Then the random variables $S_{i, n}=T_{i, n}-T_{i, n-1}$ are independent and identically distributed with continuous cumulative distribution function (CDF) $F_{i}$. The weights of the fish caught at location $i$ are given by a sequence of independent and identically distributed random variables $X_{i, 0}, X_{i, 1}, X_{i, 2}, \ldots$ with CDF $H_{i}$ (we fix $X_{1,0}=0$ and $X_{2,0}=0$ ). The renewal process is independent of the order in which the fish are caught. The angler's success is defined as the difference between the utility function $g_{i}:[0, \infty) \rightarrow\left[0, G_{i}\right]$, which is dependent on the size of the fish caught, and the time-dependent cost function $c_{i}:\left[0, t_{0}\right] \rightarrow\left[0, C_{i}\right]$. We assume that $g_{i}$ and $c_{i}$ are continuous and bounded functions, and, in addition, that $c_{i}$ is differentiable. The utility function and the cost function differ at each location. In this way, based on the opinion of the angler, each location can be modeled. For example, the angler may consider one of the locations to yield a higher probability of catching the 'best' fish or to have a more

Received 3 April 2008; revision received 25 January 2009.

* Postal address: Wrocław University of Technology, Institute of Mathematics and Computer Science, Wybrzeże Wyspiańskiego 27, 50-370 Wrocław, Poland. Email address: anna.karpowicz@pwr.wroc.pl 
comfortable pier. The mass of the fish caught up to time $t$ by an angler who changes location at time $s$ is given by $M_{t}^{s}=\sum_{n=1}^{N_{1}(s \wedge t)} X_{1, n}+\sum_{n=1}^{N_{2}\left((t-s)^{+}\right)} X_{2, n}$, where $a \wedge b=\min \{a, b\}$ and $(a)^{+}=\max \{a, 0\}$. Let $Z(s, t)$ denote the angler's payoff for stopping at time $t$ if he/she changes location at time $s$. The payoff can be expressed as

$$
Z(s, t)= \begin{cases}g_{1}\left(M_{t}\right)-c_{1}(t) & \text { if } t<s \leq t_{0}, \\ g_{1}\left(M_{s}\right)-c_{1}(s)+g_{2}\left(M_{t}^{s}-M_{s}\right)-c_{2}(t-s) & \text { if } s \leq t \leq t_{0}, \\ -C & \text { if } t_{0}<t,\end{cases}
$$

where $C=C_{1}+C_{2}$. With the notation $w_{2}(m, s, \tilde{m}, t)=w_{1}(m, s)+g_{2}(\tilde{m}-m)-c_{2}(t-s)$ and $w_{1}(m, t)=g_{1}(m)-c_{1}(t),(1)$ reduces to

$$
Z(s, t)=\mathbf{1}_{\left\{t<s \leq t_{0}\right\}} w_{1}\left(M_{t}, t\right)+\mathbf{1}_{\left\{s \leq t \leq t_{0}\right\}} w_{2}\left(M_{s}, s, M_{t}^{s}, t\right)-\mathbf{1}_{\left\{t_{0}<t\right\}} C .
$$

The extension considered here is motivated by the natural, more precise models of known real applications of the fishing problem. The typical process of software testing consists of checking subroutines. The consecutive stopping times are moments when the expert stops testing one module and starts checking another module. Similarly, in proofreading the natural parts for corrections are chapters or volumes. The consecutive stopping times are moments when the proofreading process switches from one part to another.

\section{The optimization problem}

Let $\mathcal{F}_{t}=\sigma\left(X_{1,0}, T_{1,0}, X_{1,1}, T_{1,1}, \ldots, X_{1, N_{1}(t)}, T_{1, N_{1}(t)}\right)$ denote the $\sigma$-field generated by all events up to time $t$ if the parameters remain unchanged, and let $\mathcal{F}_{s, t}=\sigma\left(\mathcal{F}_{s}, X_{2,0}\right.$, $\left.T_{2,0}, \ldots, X_{2, N_{2}(t-s)}, T_{2, N_{2}(t-s)}\right), s \leq t$, denote the $\sigma$-field generated by all events up to time $t$ if the parameters change at time $s$. For simplicity of notation, we set $\mathcal{F}_{n}:=\mathcal{F}_{T_{1, n}}$ and $\mathcal{F}_{s, n}:=\mathcal{F}_{s, T_{2, n}}$. We will let $\mathcal{M}\left(\mathcal{F}_{n}\right)$ denote the set of nonnegative and $\mathcal{F}_{n}$-measurable random variables. From now on, $\mathcal{T}$ and $\mathcal{T}^{s}$ stand for the sets of stopping times with respect to the $\sigma$-fields $\left\{\mathcal{F}_{t}, t \geq 0\right\}$ and $\left\{\mathcal{F}_{s, t}, 0 \leq s \leq t\right\}$, respectively. Furthermore, define the sets $\mathcal{T}_{n, K}=\left\{\tau \in \mathcal{T}: \tau \geq 0, T_{1, n} \leq \tau \leq T_{1, K}\right\}$ and $\mathcal{T}_{n, K}^{s}=\left\{\tau \in \mathcal{T}^{s}: 0 \leq s \leq \tau, T_{2, n} \leq \tau \leq\right.$ $T_{2, K}$ \} for $n=0,1, \ldots, K$. Obviously, the angler wants to have as much success as possible before his/her fishing ticket expires. Therefore, the angler's goal is to find two optimal stopping times $\tau_{1}^{*}$ and $\tau_{2}^{*}$ such that the expected gain is maximized:

$$
\mathrm{E}\left[Z\left(\tau_{1}^{*}, \tau_{2}^{*}\right)\right]=\sup _{\tau_{1} \in \mathcal{T}} \sup _{\tau_{2} \in \mathcal{T} \tau_{1}} \mathrm{E}\left[Z\left(\tau_{1}, \tau_{2}\right)\right]
$$

where $\tau_{1}^{*}$ is the time when the angler should change location and $\tau_{2}^{*}$ is the time when the angler should stop fishing. These stopping times should be less than the fixed fishing time, $t_{0}$. The process $Z(s, t)$ is piecewise-deterministic and belongs to the class of semi-Markov processes. The optimal stopping of similar processes was studied in [1]. We use dynamic programming methods to find these two optimal stopping times and to specify the expected success of the angler. The methods used to obtain the solution are similar to those of [7]. Let us first observe that by the properties of the conditional expectation we have

$$
\mathrm{E}\left[Z\left(\tau_{1}^{*}, \tau_{2}^{*}\right)\right]=\sup _{\tau_{1} \in \mathcal{T}} \mathrm{E}\left[\mathrm{E}\left[Z\left(\tau_{1}, \tau_{2}^{*}\right) \mid \mathcal{F}_{\tau_{1}}\right]\right]=\sup _{\tau_{1} \in \mathcal{T}} \mathrm{E}\left[J\left(\tau_{1}\right)\right]
$$

where

$$
J(s)=\mathrm{E}\left[Z\left(s, \tau_{2}^{*}\right) \mid \mathcal{F}_{s}\right]=\underset{\tau_{2} \in \mathcal{T}^{s}}{\operatorname{ess} \sup } \mathrm{E}\left[Z\left(s, \tau_{2}\right) \mid \mathcal{F}_{s}\right]
$$


Therefore, in order to find $\tau_{1}^{*}$ and $\tau_{2}^{*}$, we have to calculate $J(s)$ first. The process $J(s)$ corresponds to the value of the revenue function in the single stopping problem if the observation starts at time $s$.

\section{Construction of the second optimal stopping time}

In this section we find the solution to the single stopping problem defined by (3). We first solve the problem for a fixed number of catches, and then we consider the case in which an infinite number of fish are caught. In this section we fix $s$, the time when the angler changes location, and $m=M_{s}$, the mass of the fish caught at time $s$.

\subsection{A fixed number of catches}

In this subsection we look for an optimal stopping time $\tau_{2,0, K}^{*}:=\tau_{2, K}^{*}$ such that

$$
\mathrm{E}\left[Z\left(s, \tau_{2, K}^{*}\right) \mid \mathcal{F}_{s}\right]=\underset{\tau \in \mathcal{T}_{0, K}^{s}}{\operatorname{ess} \sup } \mathrm{E}\left[Z(s, \tau) \mid \mathcal{F}_{s}\right],
$$

where $s \geq 0$ is the (fixed) time when the angler changes location and $K$ is the maximum number of catches which can occur. Let us define, for $n=K, \ldots, 1,0$,

$$
\Gamma_{n, K}^{s}=\underset{\tau \in \mathcal{T}_{n, K}^{s}}{\operatorname{ess} \sup } \mathrm{E}\left[Z(s, \tau) \mid \mathcal{F}_{s, n}\right]=\mathrm{E}\left[Z\left(s, \tau_{2, n, K}^{*}\right) \mid \mathcal{F}_{s, n}\right]
$$

and observe that $\Gamma_{K, K}^{s}=Z\left(s, T_{2, K}\right)$. The following lemma (see [2, p. 308]) plays a crucial role in our subsequent considerations.

Lemma 1. If $\tau_{1} \in \mathcal{T}$ and $\tau_{2} \in \mathcal{T}^{s}$, then there exist $R_{1, n} \in \mathcal{M}\left(\mathcal{F}_{n}\right)$ and $R_{2, n} \in \mathcal{M}\left(\mathcal{F}_{s, n}\right)$, respectively, such that $\tau_{i} \wedge T_{i, n+1}=\left(T_{i, n}+R_{i, n}\right) \wedge T_{i, n+1}$ on $\left\{\tau_{i} \geq T_{i, n}\right\}, i=1,2$, almost surely (a.s.).

Now we derive the dynamic programming equations satisfied by $\Gamma_{n, K}^{s}$. To simplify the notation, we write $M_{t}=M_{t}^{s}$ for $t \leq s, M_{n}=M_{T_{1, n}}, M_{n}^{s}=M_{T_{2, n}}^{s}$, and $\bar{F}_{i}=1-F_{i}$.

Theorem 1. Let $s \geq 0$ be the time when the angler changes location. For $n=K-1, K-$ $2, \ldots, 0$,

$$
\begin{aligned}
\Gamma_{K, K}^{s} & =Z\left(s, T_{2, K}\right), \\
\Gamma_{n, K}^{s} & =\operatorname{Rss}_{R_{2, n} \in \mathcal{M}\left(\mathcal{F}_{s, n}\right)} \vartheta_{n, K}\left(M_{s}, s, M_{n}^{s}, T_{2, n}, R_{2, n}\right) \quad \text { a.s. },
\end{aligned}
$$

where

$$
\begin{aligned}
\vartheta_{n, K}(m, s, \widetilde{m}, t, r)=\mathbf{1}_{\left\{t \leq t_{0}\right\}}( & \bar{F}_{2}(r)\left[\mathbf{1}_{\left\{r \leq t_{0}-t\right\}} w_{2}(m, s, \widetilde{m}, t+r)-C \mathbf{1}_{\left\{r>t_{0}-t\right\}}\right] \\
+ & \left.\mathrm{E}\left[\mathbf{1}_{\left\{S_{2, n+1} \leq r\right\}} \Gamma_{n+1, K}^{s} \mid \mathcal{F}_{s, n}\right]\right)-C \mathbf{1}_{\left\{t>t_{0}\right\}} .
\end{aligned}
$$

Proof. First observe that the form of $\Gamma_{n, K}^{s}$ for the case in which $T_{2, n}>t_{0}$ is obvious from (1) and (4). By Lemma 1 , for $\tau \geq T_{2, n}$, we have

$$
\left\{\tau<T_{2, n+1}\right\}=\left\{\tau \wedge T_{2, n+1}<T_{2, n+1}\right\}=\left\{T_{2, n}+R_{2, n}<T_{2, n+1}\right\},
$$

and this gives

$$
\left\{\tau<T_{2, n+1}\right\}=\left\{S_{2, n+1}>R_{2, n}\right\}, \quad\left\{\tau \geq T_{2, n+1}\right\}=\left\{S_{2, n+1} \leq R_{2, n}\right\} .
$$


Assume that $T_{2, K-1} \leq t_{0}$ and take any $\tau \in \mathcal{T}_{K-1, K}^{S}$. According to Lemma 1 and (5) we obtain

$$
\begin{aligned}
\mathrm{E}\left[Z(s, \tau) \mid \mathcal{F}_{s, K-1}\right]= & \mathrm{E}\left[Z\left(s,\left(T_{2, K-1}+R_{2, K-1}\right) \wedge T_{2, K}\right) \mid \mathcal{F}_{s, K-1}\right] \\
= & \mathrm{E}\left[\mathbf{1}_{\left\{S_{2, K} \leq R_{2, K-1}\right\}} Z\left(s, T_{2, K}\right) \mid \mathcal{F}_{s, K-1}\right] \\
& +\mathrm{E}\left[\mathbf{1}_{\left\{S_{2, K}>R_{2, K-1}\right\}} Z\left(s, T_{2, K-1}+R_{2, K-1}\right) \mid \mathcal{F}_{s, K-1}\right] .
\end{aligned}
$$

From (2), and by the definition of $\Gamma_{K, K}^{s}$, we conclude that, for $\tau \in \mathcal{T}_{K-1, K}^{s}$,

$$
\begin{aligned}
\mathrm{E}\left[Z(s, \tau) \mid \mathcal{F}_{s, K-1}\right]= & \left(\mathbf{1}_{\left\{R_{2, K-1} \leq t_{0}-T_{2, K-1}\right\}} w_{2}\left(M_{s}, s, M_{K-1}^{s}, T_{2, K-1}+R_{2, K-1}\right)\right. \\
& \left.-C \mathbf{1}_{\left\{R_{2, K-1}>t_{0}-T_{2, K-1}\right\}}\right) \bar{F}_{2}\left(R_{2, K-1}\right) \\
& +\mathrm{E}\left[\mathbf{1}_{\left\{S_{2, K} \leq R_{2, K-1}\right\}} \Gamma_{K, K}^{s} \mid \mathcal{F}_{s, K-1}\right] .
\end{aligned}
$$

Now suppose that $T_{2, n-1} \leq t_{0}$ and that the lemma holds for some $n=1, \ldots, K-1$. Let us take any $\tau \in \mathcal{T}_{n-1, K}^{s}$. According to (5) and the properties of the conditional expectation,

$$
\begin{aligned}
\mathrm{E}\left[Z(s, \tau) \mid \mathcal{F}_{s, n-1}\right]= & \mathrm{E}\left[\mathbf{1}_{\left\{S_{2, n} \leq R_{2, n-1}\right\}} \mathrm{E}\left[Z\left(s, \tau \vee T_{2, n}\right) \mid \mathcal{F}_{s, n}\right] \mid \mathcal{F}_{s, n-1}\right] \\
& +\mathrm{E}\left[\mathbf{1}_{\left\{S_{2, n}>R_{2, n-1}\right\}} Z\left(s, \tau \wedge T_{2, n}\right) \mid \mathcal{F}_{s, n-1}\right],
\end{aligned}
$$

where $a \vee b=\max \{a, b\}$. Note that, by Lemma 1 ,

$\mathrm{E}\left[\mathbf{1}_{\left\{S_{2, n}>R_{2, n-1}\right\}} Z\left(s, \tau \wedge T_{2, n}\right) \mid \mathcal{F}_{s, n-1}\right]=\mathrm{E}\left[\mathbf{1}_{\left\{S_{2, n}>R_{2, n-1}\right\}} Z\left(s, T_{2, n-1}+R_{2, n-1}\right) \mid \mathcal{F}_{s, n-1}\right]$, and this clearly forces

$$
\begin{aligned}
\mathrm{E}\left[Z(s, \tau) \mid \mathcal{F}_{s, n-1}\right]= & \mathrm{E}\left[\mathbf{1}_{\left\{S_{2, n} \leq R_{2, n-1}\right\}} \mathrm{E}\left[Z\left(s, \tau \vee T_{2, n}\right) \mid \mathcal{F}_{s, n}\right] \mid \mathcal{F}_{s, n-1}\right] \\
& +\bar{F}_{2}\left(R_{2, n-1}\right)\left(\mathbf{1}_{\left\{R_{2, n-1} \leq t_{0}-T_{2, n-1}\right\}} w_{2}\left(M_{s}, s, M_{n-1}^{s}, T_{2, n-1}+R_{2, n-1}\right)\right. \\
& \left.-C \mathbf{1}_{\left\{R_{2, n-1}>t_{0}-T_{2, n-1}\right\}}\right) .
\end{aligned}
$$

Since $\tau \vee T_{2, n} \in \mathcal{T}_{n, K}^{s}$, we apply the induction hypothesis and obtain

$$
\begin{aligned}
\mathrm{E}\left[Z(s, \tau) \mid \mathcal{F}_{s, n-1}\right] \leq & \mathrm{E}\left[\mathbf{1}_{\left\{S_{2, n} \leq R_{2, n-1}\right\}} \Gamma_{n, K}^{s} \mid \mathcal{F}_{s, n-1}\right] \\
& +\bar{F}_{2}\left(R_{2, n-1}\right)\left(\mathbf{1}_{\left\{R_{2, n-1} \leq t_{0}-T_{2, n-1}\right\}} w_{2}\left(M_{s}, s, M_{n-1}^{s}, T_{2, n-1}+R_{2, n-1}\right)\right. \\
& \left.\quad-C \mathbf{1}_{\left\{R_{2, n-1}>t_{0}-T_{2, n-1}\right\}}\right) \\
& \leq \underset{R_{2, n-1} \in \mathcal{M}\left(\mathcal{F}_{s, n-1}\right)}{\operatorname{ess~sup}} \vartheta_{n-1, K}\left(M_{s}, s, M_{n-1}^{s}, T_{2, n-1}, R_{2, n-1}\right) \quad \text { a.s. }
\end{aligned}
$$

This gives

$$
\Gamma_{n-1, K}^{s} \leq \operatorname{ess}_{R_{2, n-1} \in \mathcal{M}\left(\widetilde{\mathcal{F}}_{s, n-1}\right)} \vartheta_{n-1, K}\left(M_{s}, s, M_{n-1}^{s}, T_{2, n-1}, R_{2, n-1}\right) \quad \text { a.s. }
$$

Let us define, for any $R_{2, n}$, the stopping time

$$
\sigma= \begin{cases}\tau_{2, n, K}^{*} & \text { if } R_{2, n-1} \geq S_{2, n}, \\ T_{2, n-1}+R_{2, n-1} & \text { if } R_{2, n-1}<S_{2, n},\end{cases}
$$

where $\tau_{2, n, K}^{*}$ is such that $\Gamma_{n, K}^{s}=\mathrm{E}\left[Z\left(s, \tau_{2, n, K}^{*}\right) \mid \mathcal{F}_{s, n}\right]$. We conclude from (4) that $\Gamma_{n-1, K}^{s} \geq$ $\mathrm{E}\left[Z(s, \sigma) \mid \mathcal{F}_{s, n-1}\right]$ for all $R_{2, n-1} \in \mathcal{M}\left(\mathcal{F}_{s, n-1}\right)$, and it follows that

$$
\Gamma_{n-1, K}^{s} \geq \operatorname{ess~sup}_{R_{2, n-1} \in \mathcal{M}\left(\widetilde{F}_{s, n-1}\right)} \vartheta_{n-1, K}\left(M_{s}, s, M_{n-1}^{s}, T_{2, n-1}, R_{2, n-1}\right),
$$

which completes the proof. 
The following remark results from the analytical properties of the function $\vartheta_{n, K}$.

Remark 1. There exists an $R_{2, n}^{*}$ such that $\Gamma_{n, K}^{s}=\vartheta_{n, K}\left(M_{s}, s, M_{n}^{s}, T_{2, n}, R_{2, n}^{*}\right)$.

Theorem 2. Let $\left\{R_{2, i}^{*}\right\}_{i=0}^{K} \in \mathcal{M}\left(\mathcal{F}_{s, i}\right)$ for every $i=0,1, \ldots, K$, and let $R_{2, K}^{*}=0$. Moreover, for $n=0, \ldots, K$, set $\eta_{n, K}^{s}=K \wedge \inf \left\{i \geq n: R_{2, i}^{*}<S_{2, i+1}\right\}$. Then $\Gamma_{n, K}^{s}=$ $\mathrm{E}\left[Z\left(s, \tau_{2, n, K}^{*}\right) \mid \mathcal{F}_{s, n}\right]$, where $\tau_{2, n, K}^{*}=T_{2, \eta_{n, K}^{s}}+R_{2, \eta_{n, K}^{s}}^{*}$.

Proof. Note that $\tau_{2, n, K}^{*}$ can be expressed as

$$
\tau_{2, n, K}^{*}= \begin{cases}\tau_{2, n+1, K}^{*} & \text { if } R_{2, n}^{*} \geq S_{2, n+1}, \\ T_{2, n}+R_{2, n}^{*} & \text { if } R_{2, n}^{*}<S_{2, n+1},\end{cases}
$$

and $\tau_{2, K, K}^{*}=T_{2, K}$. Let us check the backward induction hypothesis for $n=K-1$. From (7), carrying out similar calculations to those in the proof of Theorem 1, we obtain

$$
\mathrm{E}\left[Z\left(s, \tau_{2, K-1, K}^{*}\right) \mid \mathcal{F}_{s, K-1}\right]=\mathrm{E}\left[Z\left(s,\left(T_{2, K-1}+R_{2, K-1}^{*}\right) \wedge T_{2, K}\right) \mid \mathcal{F}_{s, K-1}\right]=\Gamma_{K-1, K}^{s} .
$$

Now assume that $\mathrm{E}\left[Z\left(s, \tau_{2, n, K}^{*}\right) \mid \mathcal{F}_{s, n}\right]=\Gamma_{n, K}^{s}$ for some $n=1, \ldots, K-1$. From (7),

$$
\begin{aligned}
\mathrm{E}\left[Z\left(s, \tau_{2, n-1, K}^{*}\right) \mid \mathcal{F}_{s, n-1}\right]= & \mathrm{E}\left[\mathbf{1}_{\left\{S_{2, n}>R_{2, n-1}^{*}\right\}} Z\left(s, T_{2, n-1}+R_{2, n-1}^{*}\right) \mid \mathcal{F}_{s, n-1}\right] \\
& +\mathrm{E}\left[\mathbf{1}_{\left\{S_{2, n} \leq R_{2, n-1}^{*}\right\}} Z\left(s, \tau_{2, n, K}^{*}\right) \mid \mathcal{F}_{s, n-1}\right] .
\end{aligned}
$$

Proceeding as in the proof of Theorem 1 (see (6)) and using the induction hypothesis, we find that $\mathrm{E}\left[Z\left(s, \tau_{2, n-1, K}^{*}\right) \mid \mathcal{F}_{s, n-1}\right]=\Gamma_{n-1, K}^{s}$.

Lemma 2. We have $\Gamma_{n, K}^{s}=\gamma_{K-n}^{s, M_{s}}\left(M_{n}^{s}, T_{2, n}\right)$ for $n=K, \ldots, 0$, where the sequence of functions $\gamma_{j}^{s, m}$ is given recursively as

$$
\begin{aligned}
& \gamma_{0}^{s, m}(\tilde{m}, t)=\mathbf{1}_{\left\{t \leq t_{0}\right\}} w_{2}(m, s, \tilde{m}, t)-C \mathbf{1}_{\left\{t>t_{0}\right\}}, \\
& \gamma_{j}^{s, m}(\tilde{m}, t)=\mathbf{1}_{\left\{t \leq t_{0}\right\}} \sup _{r \geq 0} \kappa_{2, \gamma_{j-1}^{s, m}}(m, s, \tilde{m}, t, r)-C \mathbf{1}_{\left\{t>t_{0}\right\}},
\end{aligned}
$$

where

$$
\begin{aligned}
\kappa_{2, \delta}(m, s, \tilde{m}, t, r)= & \bar{F}_{2}(r)\left(\mathbf{1}_{\left\{r \leq t_{0}-t\right\}} w_{2}(m, s, \tilde{m}, t+r)-C \mathbf{1}_{\left\{r>t_{0}-t\right\}}\right) \\
& +\int_{0}^{r} \mathrm{~d} F_{2}(z) \int_{0}^{\infty} \delta(\tilde{m}+x, t+z) \mathrm{d} H_{2}(x)
\end{aligned}
$$

for any function $\delta$.

Proof. As the case in which $t>t_{0}$ is obvious, we assume that $T_{2, n} \leq t_{0}$ for $n=0, \ldots, K-1$. Note that, according to Theorem $1, \Gamma_{K, K}^{s}=\gamma_{0}^{s, M_{s}}\left(M_{K}^{s}, T_{2, K}\right)$; thus, the lemma is satisfied for $n=K$. Let $n=K-1$. Then Theorem 1 and the induction hypothesis yield

$$
\begin{aligned}
\Gamma_{K-1, K}^{s}=\underset{R_{2, K-1} \in \mathcal{M}\left(\mathcal{F}_{s, K-1}\right)}{\operatorname{ess} \sup _{1}}( & \mathrm{E}\left[\mathbf{1}_{\left\{S_{2, K} \leq R_{2, K-1}\right\}} \gamma_{0}^{s, M_{s}}\left(M_{K}^{s}, T_{2, K}\right) \mid \mathcal{F}_{s, K-1}\right] \\
+ & \left\{\mathbf{1}_{\left\{R_{2, K-1} \leq t_{0}-T_{2, K-1}\right\}} w_{2}\left(M_{s}, s, M_{K-1}^{s}, T_{2, K-1}+R_{2, K-1}\right)\right. \\
& \left.\left.\quad-C \mathbf{1}_{\left\{R_{2, K-1}>t_{0}-T_{2, K-1}\right\}}\right\} \bar{F}_{2}\left(R_{2, K-1}\right)\right) \quad \text { a.s., }
\end{aligned}
$$

where $M_{K}^{s}=M_{K-1}^{s}+X_{2, K}, T_{2, K}=T_{2, K-1}+S_{2, K}$, and the random variables $X_{2, K}$ and $S_{2, K}$ are independent of $\mathcal{F}_{s, K-1}$. Moreover, the random variables $R_{2, K-1}, M_{K-1}^{s}$, and 
$T_{2, K-1}$ are $\mathcal{F}_{S, K-1}$-measurable. The expectation under the ess sup in (9) can be expressed as $\int_{0}^{R_{2, K-1}} \mathrm{~d} F_{2}(z) \int_{0}^{\infty} \gamma_{0}^{s, M_{s}}\left(M_{K-1}^{s}+x, T_{2, K-1}+z\right) \mathrm{d} H_{2}(x)$, and it follows that $\Gamma_{K-1, K}^{s}=$ $\gamma_{1}^{s, M_{s}}\left(M_{K-1}^{s}, T_{2, K-1}\right)$ a.s. Suppose that $\Gamma_{n, K}^{s}=\gamma_{K-n}^{s, M_{s}}\left(M_{n}^{s}, T_{2, n}\right)$ for any $n=1, \ldots, K-1$. Similarly to before, we conclude, by Theorem 1 and the induction hypothesis, that

$$
\begin{aligned}
\Gamma_{n-1, K}^{S} & \\
=\operatorname{Ressup}_{R_{2, n-1} \in \mathcal{M}\left(\mathcal{F}_{s, n-1}\right)}( & \left\{\mathbf{1}_{\left\{R_{2, n-1} \leq t_{0}-T_{2, n-1}\right\}} w_{2}\left(M_{s}, s, M_{n-1}^{s}, T_{2, n-1}+R_{2, n-1}\right)\right. \\
& \left.-C \mathbf{1}_{\left\{R_{2, n-1}>t_{0}-T_{2, n-1}\right\}}\right\} \bar{F}_{2}\left(R_{2, n-1}\right) \\
& \left.+\int_{0}^{R_{2, n-1}} \mathrm{~d} F_{2}(z) \int_{0}^{\infty} \gamma_{K-n}^{s, M_{s}}\left(M_{n-1}^{s}+x, T_{2, n-1}+z\right) \mathrm{d} H_{2}(x)\right) \text { a.s.; }
\end{aligned}
$$

therefore, $\Gamma_{n-1, K}^{s}=\gamma_{K-(n-1)}^{s, M_{s}}\left(M_{n-1}^{s}, T_{2, n-1}\right)$.

From now on, we will use $\alpha_{i}$ to denote the hazard rate of $F_{i}$ (i.e. $\alpha_{i}=f_{i} / \bar{F}_{i}$ ) and, for notational ease, set $\Delta_{i}(a)=\mathrm{E}\left[g_{i}\left(a+X_{i}\right)-g_{i}(a)\right]$.

Proposition 1. For $j=0,1, \ldots, K$, the sequence of functions $\gamma_{j}^{s, m}$ can be expressed as

$$
\gamma_{j}^{s, m}(\tilde{m}, t)=\mathbf{1}_{\left\{t \leq t_{0}\right\}}\left(w_{2}(m, s, \tilde{m}, t)+y_{2, j}\left(\tilde{m}-m, t-s, t_{0}-t\right)\right)-C \mathbf{1}_{\left\{t>t_{0}\right\}},
$$

where $y_{2, j}(a, b, c)$ is given recursively as

$$
y_{2,0}(a, b, c)=0, \quad y_{2, j}(a, b, c)=\max _{0 \leq r \leq c} \phi_{2, y_{2, j-1}}(a, b, c, r),
$$

and

$$
\phi_{2, \delta}(a, b, c, r)=\int_{0}^{r} \bar{F}_{2}(z)\left(\alpha_{2}(z)\left\{\Delta_{2}(a)+\mathrm{E}\left[\delta\left(a+X_{2}, b+z, c-z\right)\right]\right\}-c_{2}^{\prime}(b+z)\right) \mathrm{d} z .
$$

Proof. Clearly,

$$
\int_{0}^{r} \mathrm{~d} F_{2}(z) \int_{0}^{\infty} \gamma_{j-1}^{s, m}(\tilde{m}+x, t+z) \mathrm{d} H_{2}(x)=\mathrm{E}\left[\mathbf{1}_{\left\{S_{2} \leq r\right\}} \gamma_{j-1}^{s, m}\left(\tilde{m}+X_{2}, t+S_{2}\right)\right],
$$

where $S_{2}$ has $\mathrm{CDF} F_{2}$ and $X_{2}$ has $\mathrm{CDF} H_{2}$. Since $\kappa_{2, \gamma} s, m(m, s, \tilde{m}, t, r)$ is continuous for $r \in\left[0, t_{0}-t\right]$, the supremum in (8) can be changed into a maximum. Let $r>t_{0}-t$. Then

$$
\begin{aligned}
\kappa_{2, \gamma_{j-1}^{s, m}}(m, s, \tilde{m}, t, r)= & \mathrm{E}\left[\mathbf{1}_{\left\{S_{2} \leq t_{0}-t\right\}} \gamma_{j-1}^{s, m}\left(\tilde{m}+X_{2}, t+S_{2}\right)\right]-C \bar{F}_{2}\left(t_{0}-t\right) \\
\leq & \mathrm{E}\left[\mathbf{1}_{\left\{S_{2} \leq t_{0}-t\right\}} \gamma_{j-1}^{s, m}\left(\tilde{m}+X_{2}, t+S_{2}\right)\right] \\
& +\bar{F}_{2}\left(t_{0}-t\right) w_{2}\left(m, s, \tilde{m}, t_{0}\right) \\
= & \kappa_{2, \gamma_{j-1}^{s, m}}\left(m, s, \tilde{m}, t, t_{0}-t\right) .
\end{aligned}
$$

The above calculations yield, for $j=0,1, \ldots, K$,

$$
\gamma_{j}^{s, m}(\tilde{m}, t)=\mathbf{1}_{\left\{t \leq t_{0}\right\}} \max _{0 \leq r \leq t_{0}-t} \varphi_{j}(m, s, \tilde{m}, t, r)-C \mathbf{1}_{\left\{t>t_{0}\right\}}
$$

where $\varphi_{j}(m, s, \tilde{m}, t, r)=\bar{F}_{2}(r) w_{2}(m, s, \tilde{m}, t+r)+\mathrm{E}\left[\mathbf{1}_{\left\{S_{2} \leq r\right\}} \gamma_{j-1}^{s, m}\left(\tilde{m}+X_{2}, t+S_{2}\right)\right]$. We have $t+z \leq t_{0}$ for $z \leq r, r \leq t_{0}-t$, and for this reason we can consider the cases in which 
$t \leq t_{0}$ and $t>t_{0}$ separately. Consider the $t \leq t_{0}$ case. Then $\gamma_{0}^{s, m}(\tilde{m}, t)=w_{2}(m, s, \tilde{m}, t)$ and the hypothesis is true for $j=0$. The task is now to calculate $\gamma_{j+1}^{s, m}(\tilde{m}, t)$ given $\gamma_{j}^{s, m}(\cdot, \cdot)$. The induction hypothesis implies that, for $t \leq t_{0}$,

$$
\begin{aligned}
\varphi_{j+1}(m, s, \tilde{m}, t, r)= & \bar{F}_{2}(r) w_{2}(m, s, \tilde{m}, t+r)+\mathrm{E}\left[\mathbf{1}_{\left\{S_{2} \leq r\right\}} \gamma_{j}^{s, m}\left(\tilde{m}+X_{2}, t+S_{2}\right)\right] \\
= & g_{1}(m)-c_{1}(s)+\bar{F}_{2}(r)\left(g_{2}(\tilde{m}-m)-c_{2}(t-s+r)\right) \\
& +\int_{0}^{r} f_{2}(z)\left(\mathrm{E}\left[g_{2}\left(\tilde{m}-m+X_{2}\right)\right]-c_{2}(t-s+z)\right. \\
& \left.\quad+\mathrm{E}\left[y_{2, j}\left(\tilde{m}-m+X_{2}, t-s+z, t_{0}-t-z\right)\right]\right) \mathrm{d} z .
\end{aligned}
$$

It is clear that, for any $a$ and $b$,

$$
\begin{aligned}
\bar{F}_{2}(r)\left(g_{2}(a)-c_{2}(b+r)\right)= & g_{2}(a)-c_{2}(b) \\
& -\int_{0}^{r}\left(f_{2}(z)\left[g_{2}(a)-c_{2}(b+z)\right]+\bar{F}_{2}(z) c_{2}^{\prime}(b+z)\right) \mathrm{d} z ;
\end{aligned}
$$

therefore,

$$
\begin{aligned}
& \varphi_{j+1}(m, s, \tilde{m}, t, r) \\
& =w_{2}(m, s, \tilde{m}, t)+\int_{0}^{r} \bar{F}_{2}(z)\left(-c_{2}^{\prime}(t-s+z)\right. \\
& +\alpha_{2}(z)\left\{\Delta_{2}(\tilde{m}-m)\right. \\
& \left.\left.+\mathrm{E}\left[y_{2, j}\left(\tilde{m}-m+X_{2}, t-s+z, t_{0}-t-z\right)\right]\right\}\right) \mathrm{d} z .
\end{aligned}
$$

The case in which $t>t_{0}$ is trivial. This completes the proof.

Following the approach of [4], we find a second optimal stopping time. Let $B=B([0, \infty) \times$ $\left.\left[0, t_{0}\right] \times\left[0, t_{0}\right]\right)$ be the space of all bounded, continuous functions with the norm $\|\delta\|=$ $\sup _{a, b, c}|\delta(a, b, c)|$. It is easy to check that $B$ equipped with the supremum norm is a complete space. The operator $\Phi_{2}: B \rightarrow B$ is defined by

$$
\left(\Phi_{2} \delta\right)(a, b, c)=\max _{0 \leq r \leq c} \phi_{2, \delta}(a, b, c, r) .
$$

Let us observe that $y_{2, j}(a, b, c)=\left(\Phi_{2} y_{2, j-1}\right)(a, b, c)$. Remark 1 now implies that there exists a function $r_{2, j}^{*}$ such that $y_{2, j}(a, b, c)=\phi_{2, y_{2, j-1}}\left(a, b, c, r_{2, j}^{*}(a, b, c)\right)$, and this gives

$$
\begin{gathered}
\gamma_{j}^{s, m}(\tilde{m}, t)=\mathbf{1}_{\left\{t \leq t_{0}\right\}}\left(\phi_{2, y_{2, j-1}}\left(\tilde{m}-m, t-s, t_{0}-t, r_{2, j}^{*}\left(\tilde{m}-m, t-s, t_{0}-t\right)\right)\right. \\
\left.+w_{2}(m, s, \tilde{m}, t)\right)-C \mathbf{1}_{\left\{t>t_{0}\right\}} .
\end{gathered}
$$

The following theorem, which determines the optimal stopping times $\tau_{2, n, K}^{*}$, is a consequence of the above considerations.

Theorem 3. Let $R_{2, i}^{*}=r_{2, K-i}^{*}\left(M_{i}^{s}-M_{s}, T_{2, i}-s, t_{0}-T_{2, i}\right)$ for $i=0,1, \ldots, K$. Moreover, let $\eta_{n, K}^{s}=K \wedge \inf \left\{i \geq n: R_{2, i}^{*}<S_{2, i+1}\right\}$. Then the stopping time $\tau_{2, n, K}^{*}=T_{2, \eta_{n, K}^{s}}+R_{2, \eta_{n, K}^{s}}^{*}$ is optimal in the class $\mathcal{T}_{n, K}^{s}$ and $\Gamma_{n, K}^{s}=\mathrm{E}\left[Z\left(s, \tau_{2, n, K}^{*}\right) \mid \mathcal{F}_{s, n}\right]$. 


\subsection{An infinite number of catches}

The task is now to find the function $J(s)$ and the stopping time $\tau_{2}^{*}$ that is optimal in the $\mathcal{T}^{s}$ class. In order to obtain the solution to the single stopping problem for an infinite number of catches, it is necessary to set the restriction that $F_{2}\left(t_{0}\right)<1$.

Lemma 3. If $F_{2}\left(t_{0}\right)<1$ then the operator $\Phi_{2}: B \rightarrow B$ defined in $(10)$ is a contraction.

Proof. Let $\delta_{i} \in B$ for $i=1,2$. It is easily seen that there exists a $\rho_{i}$ such that

$$
\left(\Phi_{2} \delta_{i}\right)(a, b, c)=\phi_{2, \delta_{i}}\left(a, b, c, \rho_{i}\right) .
$$

Thus, we obtain

$$
\begin{aligned}
\left(\Phi_{2} \delta_{1}\right)(a, b, c)-\left(\Phi_{2} \delta_{2}\right)(a, b, c) & =\phi_{2, \delta_{1}}\left(a, b, c, \rho_{1}\right)-\phi_{2, \delta_{2}}\left(a, b, c, \rho_{2}\right) \\
& \leq \phi_{2, \delta_{1}}\left(a, b, c, \rho_{1}\right)-\phi_{2, \delta_{2}}\left(a, b, c, \rho_{1}\right) \\
& =\int_{0}^{\rho_{1}} \mathrm{~d} F_{2}(z) \int_{0}^{\infty}\left(\delta_{1}-\delta_{2}\right)(a+x, b+z, c-s) \mathrm{d} H_{2}(x) \\
& \leq \int_{0}^{\rho_{1}} \mathrm{~d} F_{2}(z) \int_{0}^{\infty} \sup _{a, b, c}\left|\left(\delta_{1}-\delta_{2}\right)(a, b, c)\right| \mathrm{d} H_{2}(x) \\
& \leq F_{2}\left(\rho_{1}\right)\left\|\delta_{1}-\delta_{2}\right\| \\
& \leq \sigma\left\|\delta_{1}-\delta_{2}\right\|,
\end{aligned}
$$

where $\sigma=F_{2}\left(t_{0}\right)$. Similarly to before, $\left(\Phi_{2} \delta_{2}\right)(a, b, c)-\left(\Phi_{2} \delta_{1}\right)(a, b, c) \leq \sigma\left\|\delta_{2}-\delta_{1}\right\|$. Finally, we conclude that $\left\|\Phi_{2} \delta_{1}-\Phi_{2} \delta_{2}\right\| \leq \sigma\left\|\delta_{1}-\delta_{2}\right\|$, where $\sigma \in[0,1)$.

Remark 2. Applying Remark 1, Lemma 3, and the fixed point theorem we conclude that there exists a $y_{2} \in B$ such that $y_{2}=\Phi_{2} y_{2}$ and $\lim _{K \rightarrow \infty}\left\|y_{2, K}-y_{2}\right\|=0$.

According to Remark 2, $y_{2}$ is a uniform limit of $y_{2, K}$ when $K$ tends to $\infty$, which implies that $y_{2}$ is measurable and $\gamma^{s, m}=\lim _{K \rightarrow \infty} \gamma_{K}^{s, m}$ is given by

$$
\gamma^{s, m}(\tilde{m}, t)=\mathbf{1}_{\left\{t \leq t_{0}\right\}}\left(w_{2}(m, s, \tilde{m}, t)+y_{2}\left(\tilde{m}-m, t-s, t_{0}-t\right)\right)-C \mathbf{1}_{\left\{t>t_{0}\right\}} .
$$

We now calculate the optimal strategy and the expected gain for an angler who has changed fishing location.

Theorem 4. If $F_{2}\left(t_{0}\right)<1$, with density function $f_{2}$, then

(i) for $n \in \mathbb{N}$, the limit $\tau_{2, n}^{*}=\lim _{K \rightarrow \infty} \tau_{2, n, K}^{*}$ a.s. exists and $\tau_{2, n}^{*} \leq t_{0}$ is an optimal stopping rule in the set $\mathcal{T}^{s} \cap\left\{\tau \geq T_{2, n}\right\}$,

(ii) $\mathrm{E}\left[Z\left(s, \tau_{2, n}^{*}\right) \mid \mathcal{F}_{s, n}\right]=\gamma^{s, m}\left(M_{n}^{s}, T_{2, n}\right)$ a.s.

Proof. (i) Let us first prove the existence of $\tau_{2, n}^{*}$. By the definition of $\Gamma_{n, K+1}^{s}$ we have

$$
\begin{aligned}
& \Gamma_{n, K+1}^{s}=\underset{\tau \in \mathcal{T}_{n, K}^{s}}{\operatorname{ess} \sup } \mathrm{E}\left[Z(s, \tau) \mid \mathcal{F}_{s, n}\right] \vee \underset{\tau \in \mathcal{T}_{K, K+1}^{s}}{\operatorname{esssup}} \mathrm{E}\left[Z(s, \tau) \mid \mathcal{F}_{s, n}\right] \\
& =\mathrm{E}\left[Z\left(s, \tau_{2, n, K}^{*}\right) \mid \mathcal{F}_{s, n}\right] \vee \mathrm{E}\left[Z\left(s, \sigma^{*}\right) \mid \mathcal{F}_{s, n}\right] ;
\end{aligned}
$$

thus, we observe that $\tau_{2, n, K+1}^{*}$ is equal to $\tau_{2, n, K}^{*}$ or $\sigma^{*}$, where $\tau_{2, n, K}^{*} \in \mathcal{T}_{n, K}^{s}$ and $\sigma^{*} \in \mathcal{T}_{K, K+1}^{s}$. It follows that $\tau_{2, n, K+1}^{*} \geq \tau_{2, n, K}^{*}$, which implies that the sequence $\tau_{2, n, K}^{*}$ is nondecreasing with 
respect to $K$. Moreover, $R_{2, i}^{*} \leq t_{0}-T_{2, i}$ for all $i=0, \ldots, K$; thus, $\tau_{2, n, K}^{*} \leq t_{0}$ and, therefore, $\tau_{2, n}^{*} \leq t_{0}$ exists.

Now define the process $\xi^{s}(t)=\left(t, M_{t}^{s}, V(t)\right)$, where $s$ is fixed and $V(t)=t-T_{2, N_{2}(t)}$. Here $\xi^{s}(t)$ is a Markov process with state space $\left[s, t_{0}\right] \times[m, \infty) \times[0, \infty)$. In general, the infinitesimal operator for $\xi^{s}$ is given by

$$
\begin{aligned}
A p^{s, m}(t, \tilde{m}, v)= & \frac{\partial}{\partial t} p^{s, m}(t, \tilde{m}, v)+\frac{\partial}{\partial v} p^{s, m}(t, \tilde{m}, v) \\
& +\alpha_{2}(v)\left(\int_{\mathbb{R}^{+}} p^{s, m}(t, x, 0) \mathrm{d} H_{2}(x)-p^{s, m}(t, \tilde{m}, v)\right),
\end{aligned}
$$

where $p^{s, m}(t, \tilde{m}, v):[0, \infty) \times[0, \infty) \times[0, \infty) \rightarrow \mathbb{R}$ is continuous, bounded, and measurable, with bounded left-hand derivatives with respect to $t$ and $v$ (see [1] and [9]). Note that, for $t \geq s$, the process $Z(s, t)$ can be expressed as $Z(s, t)=p^{s, m}\left(\xi^{s}(t)\right)$, where

$$
p^{s, m}\left(\xi^{s}(t)\right)= \begin{cases}g_{1}(m)-c_{1}(s)+g_{2}\left(M_{t}^{s}-m\right)-c_{2}(t-s) & \text { if } s \leq t \leq t_{0}, \\ -C & \text { if } t_{0}<t\end{cases}
$$

It easily follows that in our case $A p^{s, m}(t, \tilde{m}, v)=0$ for $t_{0}<t$ and

$$
A p^{s, m}(t, \tilde{m}, v)=\alpha_{2}(v)\left(\mathrm{E}\left[g_{2}\left(\tilde{m}+X_{2}-m\right)\right]-g_{2}(\tilde{m}-m)\right)-c_{2}^{\prime}(t-s)
$$

for $s \leq t \leq t_{0}$. The process $p^{s, m}\left(\xi^{s}(t)\right)-p^{s, m}\left(\xi^{s}(s)\right)-\int_{s}^{t}\left(A p^{s, m}\right)\left(\xi^{s}(z)\right) \mathrm{d} z$ is a martingale with respect to $\sigma\left(\xi^{s}(z), z \leq t\right)$, which is the same as $\mathcal{F}_{s, t}$. This result can be found in [3]. Since $\tau_{2, n, K}^{*} \leq t_{0}$, applying Dynkin's formula we obtain

$$
\mathrm{E}\left[p^{s, m}\left(\xi^{s}\left(\tau_{2, n, K}^{*}\right)\right) \mid \mathcal{F}_{s, n}\right]-p^{s, m}\left(\xi^{s}\left(T_{2, n}\right)\right)=\mathrm{E}\left[\int_{T_{2, n}}^{\tau_{2, n, K}^{*}}\left(A p^{s, m}\right)\left(\xi^{s}(z)\right) \mathrm{d} z \mid \mathcal{F}_{s, n}\right] .
$$

From (11) we conclude that

$$
\begin{aligned}
\int_{T_{2, n}}^{\tau_{2, n, K}^{*}}\left(A p^{s, m}\right)\left(\xi^{s}(z)\right) \mathrm{d} z= & \left(\mathrm{E}\left[g_{2}\left(M_{n}^{s}+X_{2}-m\right)\right]-g_{2}\left(M_{n}^{s}-m\right)\right) \int_{T_{2, n}}^{\tau_{2, n, K}^{*}} \alpha_{2}\left(z-T_{2, n}\right) \mathrm{d} z \\
& -\int_{T_{2, n}}^{\tau_{2, n, K}^{*}} c_{2}^{\prime}(z-s) \mathrm{d} z .
\end{aligned}
$$

Moreover, let us check that

$$
\begin{gathered}
\left|\int_{T_{2, n}}^{\tau_{2, n, K}^{*}} \alpha_{2}\left(z-T_{2, n}\right) \mathrm{d} z\right| \leq \frac{1}{\bar{F}_{2}\left(t_{0}\right)} \int_{T_{2, n}}^{\tau_{2, n, K}^{*}} f_{2}\left(z-T_{2, n}\right) \mathrm{d} z \leq \frac{1}{\bar{F}_{2}\left(t_{0}\right)}<\infty, \\
\left|\int_{T_{2, n}}^{\tau_{2, n, K}^{*}} c_{2}^{\prime}(z-s) \mathrm{d} z\right|=\left|c_{2}\left(\tau_{2, n, K}^{*}-s\right)-c_{2}\left(T_{2, n}-s\right)\right|<\infty, \\
\left|\mathrm{E}\left[g_{2}\left(M_{n}^{s}+X_{2}-m\right)\right]-g_{2}\left(M_{n}^{s}-m\right)\right|<\infty,
\end{gathered}
$$

where the two last inequalities result from the fact that the functions $g_{2}$ and $c_{2}$ are bounded. On account of the above observation, we can use the dominated convergence theorem to obtain

$$
\lim _{K \rightarrow \infty} \mathrm{E}\left[\int_{T_{2, n}}^{\tau_{2, n, K}^{*}}\left(A p^{s, m}\right)\left(\xi^{s}(z)\right) \mathrm{d} z \mid \mathcal{F}_{s, n}\right]=\mathrm{E}\left[\int_{T_{2, n}}^{\tau_{2, n}^{*}}\left(A p^{s, m}\right)\left(\xi^{s}(z)\right) \mathrm{d} z \mid \mathcal{F}_{s, n}\right] .
$$


Since $\tau_{2, n}^{*} \leq t_{0}$, applying Dynkin's formula to the left-hand side of (13) we conclude that

$$
\mathrm{E}\left[\int_{T_{2, n}}^{\tau_{2, n}^{*}}\left(A p^{s, m}\right)\left(\xi^{s}(z)\right) \mathrm{d} z \mid \mathcal{F}_{s, n}\right]=\mathrm{E}\left[p^{s, m}\left(\xi^{s}\left(\tau_{2, n}^{*}\right)\right) \mid \mathcal{F}_{s, n}\right]-p^{s, m}\left(\xi^{s}\left(T_{2, n}\right)\right) \quad \text { a.s. }
$$

Combining (12), (13), and (14) we see that

$$
\lim _{K \rightarrow \infty} \mathrm{E}\left[p^{s, m}\left(\xi^{s}\left(\tau_{2, n, K}^{*}\right)\right) \mid \mathcal{F}_{s, n}\right]=\mathrm{E}\left[p^{s, m}\left(\xi^{s}\left(\tau_{2, n}^{*}\right)\right) \mid \mathcal{F}_{s, n}\right] \quad \text { a.s.; }
$$

hence,

$$
\lim _{K \rightarrow \infty} \mathrm{E}\left[Z\left(s, \tau_{2, n, K}^{*}\right) \mid \mathcal{F}_{s, n}\right]=\mathrm{E}\left[Z\left(s, \tau_{2, n}^{*}\right) \mid \mathcal{F}_{s, n}\right] .
$$

We now prove the optimality of $\tau_{2, n}^{*}$ in the class $\mathcal{T}^{s} \cap\left\{\tau_{2, n} \geq T_{2, n}\right\}$. Let $\tau \in \mathcal{T}^{s} \cap\left\{\tau_{2, n} \geq T_{2, n}\right\}$. It is clear that $\tau \wedge T_{2, K} \in \mathcal{T}_{n, K}^{s}$. As $\tau_{2, n, K}^{*}$ is optimal in the class $\mathcal{T}_{n, K}^{s}$, we have

$$
\lim _{K \rightarrow \infty} \mathrm{E}\left[p^{s, m}\left(\xi^{s}\left(\tau_{2, n, K}^{*}\right)\right) \mid \mathcal{F}_{s, n}\right] \geq \lim _{K \rightarrow \infty} \mathrm{E}\left[p^{s, m}\left(\xi^{s}\left(\tau \wedge T_{2, K}\right)\right) \mid \mathcal{F}_{s, n}\right] .
$$

From (15) and (16), we conclude that $\mathrm{E}\left[p^{s, m}\left(\xi^{s}\left(\tau_{2, n}^{*}\right)\right) \mid \mathcal{F}_{s, n}\right] \geq \mathrm{E}\left[p^{s, m}\left(\xi^{s}(\tau)\right) \mid \mathcal{F}_{s, n}\right]$ for any stopping time $\tau \in \mathcal{T}^{s} \cap\left\{\tau \geq T_{2, n}\right\}$, which implies that $\tau_{2, n}^{*}$ is optimal in this class.

(ii) Lemma 2 and (15) lead to $\mathrm{E}\left[Z\left(s, \tau_{2, n}^{*}\right) \mid \mathcal{F}_{s, n}\right]=\gamma^{s, M_{s}}\left(M_{n}^{s}, T_{2, n}\right)$.

The remainder of this section will be devoted to the proof of the left-hand differentiability of the function $\gamma^{s, m}(m, s)$ with respect to $s$. This property is necessary to construct the first optimal stopping time. Let $\delta(0,0, c) \in B$ be denoted by $\bar{\delta}(c)$.

Lemma 4. Let $\bar{v}(c)=\Phi_{2} \bar{\delta}(c)$, where $\bar{\delta}(c) \in B$ and $\left|\bar{\delta}_{+}^{\prime}(c)\right| \leq A_{1}, A_{1} \in \mathbb{R}^{+}$, for $c \in\left[0, t_{0}\right)$. Then $\left|\bar{v}_{+}^{\prime}(c)\right| \leq A_{2}, A_{2} \in \mathbb{R}^{+}$.

Proof. First observe that $\bar{v}_{+}^{\prime}(c)$ exists because $\bar{v}(c)=\max _{0 \leq r \leq c} \bar{\phi}_{2}(c, r)$, where $\bar{\phi}_{2}(c, r)$ is differentiable with respect to $c$ and $r$. Let us fix $h \in\left(0, t_{0}-c\right)$, and define the functions $\bar{\delta}_{1}(c)=\bar{\delta}(c+h) \in B$ and $\bar{\delta}_{2}(c)=\bar{\delta}(c) \in B$. It is obvious that $\left\|\Phi_{2} \bar{\delta}_{1}-\Phi_{2} \bar{\delta}_{2}\right\| \geq \mid \Phi_{2} \bar{\delta}_{1}(c)-$ $\Phi_{2} \bar{\delta}_{2}(c)|=| \Phi_{2} \bar{\delta}(c+h)-\Phi_{2} \bar{\delta}(c) \mid$. On the other hand, using Taylor's formula for the right-hand derivatives we obtain

$$
\left\|\bar{\delta}_{1}-\bar{\delta}_{2}\right\|=\sup _{c \in\left[0, t_{0}\right)}|\bar{\delta}(c+h)-\bar{\delta}(c)| \leq h \sup _{c \in\left[0, t_{0}\right)}\left|\bar{\delta}_{+}^{\prime}(c)\right|+|o(h)| .
$$

From the above and Lemma 3, it follows that

$$
\left|\Phi_{2} \bar{\delta}(c+h)-\Phi_{2} \bar{\delta}(c)\right|<\sigma\left\{h \sup _{c \in\left[0, t_{0}\right)}\left|\delta_{+}^{\prime}(c)\right|+|o(h)|\right\}
$$

therefore,

$$
-\sigma\left\{\sup _{c \in\left[0, t_{0}\right)}\left|\bar{\delta}_{+}^{\prime}(c)\right|+\frac{|o(h)|}{h}\right\} \leq \frac{\bar{v}(c+h)-\bar{v}(c)}{h} \leq \sigma\left\{\sup _{c \in\left[0, t_{0}\right)}\left|\bar{\delta}_{+}^{\prime}(c)\right|+\frac{|o(h)|}{h}\right\} .
$$

Letting $h \rightarrow 0^{+}$gives $\left|\bar{v}_{+}^{\prime}(c)\right| \leq \sigma A_{1}=A_{2}$.

The significance of Lemma 4 is such that the function $\bar{y}\left(t_{0}-s\right)=y_{2}\left(0,0, t_{0}-s\right)$, where $y_{2}$ was introduced in Remark 2, has bounded left-hand derivatives with respect to $s$ for $s \in\left(0, t_{0}\right]$. The following remark is an important consequence of this fact. 
Remark 3. The function $\gamma^{s, m}$ can be expressed as

$$
\gamma^{s, m}(m, s)=\mathbf{1}_{\left\{s \leq t_{0}\right\}} u(m, s)-C \mathbf{1}_{\left\{s>t_{0}\right\}},
$$

where $u(m, s)=g_{1}(m)-c_{1}(s)+g_{2}(0)-c_{2}(0)+\bar{y}_{2}\left(t_{0}-s\right)$ is continuous, bounded, and measurable, with bounded left-hand derivatives with respect to $s$.

At the end of this section, we determine the conditional value function of the second optimal stopping problem. According to (3), Theorem 4, and Remark 3, we have

$$
J(s)=\mathrm{E}\left[Z\left(s, \tau_{2}^{*}\right) \mid \mathcal{F}_{s}\right]=\gamma^{s, M_{s}}\left(M_{s}, s\right) \text { a.s. }
$$

\section{Construction of the first optimal stopping time}

In this section we formulate the solution to the double stopping problem. Let us first note that the function $u(m, s)$ has similar properties to the function $w_{2}(m, s, \tilde{m}, t)$ and that the process $J(s)$ has a similar structure to the process $Z(s, t)$. Therefore, we can proceed as in Section 3 to obtain $J(s)$. Let us again define

$$
\Gamma_{n, K}=\underset{\tau_{1} \in \mathcal{T}_{n, K}}{\operatorname{ess} \sup } \mathrm{E}\left[J\left(\tau_{1}\right) \mid \mathcal{F}_{n}\right], \quad n=K, \ldots, 1,0,
$$

which fulfills the following representation lemma.

Lemma 5. We have $\Gamma_{n, K}=\gamma_{K-n}\left(M_{n}, T_{1, n}\right)$ for $n=K, \ldots, 0$, where the sequence of functions $\gamma_{j}$ can be expressed as

$$
\gamma_{j}(m, s)=\mathbf{1}_{\left\{s \leq t_{0}\right\}}\left(u(m, s)+y_{1, j}\left(m, s, t_{0}-s\right)\right)-C \mathbf{1}_{\left\{s>t_{0}\right\}},
$$

where $y_{1, j}(a, b, c)$ is given recursively as

$$
y_{1,0}(a, b, c)=0, \quad y_{1, j}(a, b, c)=\max _{0 \leq r \leq c} \phi_{1, y_{1, j-1}}(a, b, c, r),
$$

and

$$
\begin{gathered}
\phi_{1, \delta}(a, b, c, r)=\int_{0}^{r} \bar{F}_{1}(z)\left(\alpha_{1}(z)\left[\Delta_{1}(a)+\mathrm{E}\left[\delta\left(a+X_{1}, b+z, c-z\right)\right]\right]\right. \\
\left.\left.-\bar{y}_{2-}^{\prime}(c-z)+c_{1}^{\prime}(b+z)\right)\right) \mathrm{d} z .
\end{gathered}
$$

Lemma 5 is a combination of Lemma 2 and Remark 1 from Subsection 3.1. Let the operator $\Phi_{1}: B \rightarrow B$ be defined as

$$
\left(\Phi_{1} \delta\right)(a, b, c)=\max _{0 \leq r \leq c} \phi_{1, \delta}(a, b, c, r) .
$$

Lemma 5 implies that there exists a function $r_{1, j}^{*}$ such that

$$
\gamma_{j}(m, s)=\mathbf{1}_{\left\{s \leq t_{0}\right\}}\left(u(m, s)+\phi_{1, y_{1, j-1}}\left(m, s, t_{0}-s, r_{1, j}^{*}\left(m, s, t_{0}-s\right)\right)\right)-C \mathbf{1}_{\left\{s>t_{0}\right\}} .
$$

We can now state the analogue of Theorem 3.

Theorem 5. Let $R_{1, i}^{*}=r_{1, K-i}^{*}\left(M_{i}, T_{1, i}, t_{0}-T_{1, i}\right)$ for $i=0,1, \ldots, K$. Moreover, let $\eta_{n, K}=$ $K \wedge \inf \left\{i \geq n: R_{1, i}^{*}<S_{1, i+1}\right\}$. Then $\tau_{1, n, K}^{*}=T_{1, \eta_{n, K}}+R_{1, \eta_{n, K}}^{*}$ is optimal in the class $\mathcal{T}_{n, K}$ and $\Gamma_{n, K}=\mathrm{E}\left[J\left(\tau_{1, n, K}^{*}\right) \mid \mathcal{F}_{n}\right]$. 
The following results may be proved in much the same way as the analogous theorems in Section 3.

Lemma 6. If $F_{1}\left(t_{0}\right)<1$ then the operator $\Phi_{1}: B \rightarrow B$ defined in (18) is a contraction.

Remark 4. There exists a $y_{1} \in B$ such that $y_{1}=\Phi_{1} y_{1}$ and $\lim _{K \rightarrow \infty}\left\|y_{1, K}-y_{1}\right\|=0$.

Remark 4 implies that $\gamma=\lim _{K \rightarrow \infty} \gamma_{K}$ is given by

$$
\gamma(m, s)=\mathbf{1}_{\left\{s \leq t_{0}\right\}}\left(u(m, s)+y_{1}\left(m, s, t_{0}-s\right)\right)-C \mathbf{1}_{\left\{s>t_{0}\right\}} .
$$

We can now formulate our main result.

Theorem 6. If $F_{1}\left(t_{0}\right)<1$, with density function $f_{1}$, then

(i) for $n \in \mathbb{N}$, the limit $\tau_{1, n}^{*}=\lim _{K \rightarrow \infty} \tau_{1, n, K}^{*}$ a.s. exists and $\tau_{1, n}^{*} \leq t_{0}$ is an optimal stopping rule in the set $\mathcal{T} \cap\left\{\tau \geq T_{1, n}\right\}$,

(ii) $\mathrm{E}\left[J\left(\tau_{1, n}^{*}\right) \mid \mathcal{F}_{n}\right]=\gamma\left(M_{n}, T_{1, n}\right)$ a.s.

Proof. The proof follows similarly to the proof of Theorem 4, except that the form of the infinitesimal operator is different. Define the process $\xi(s)=\left(s, M_{s}, V(s)\right)$, where $V(s)=$ $s-T_{1, N_{1}(s)}$. As before, $\xi(s)$ is a Markov process with state space $\left[0, t_{0}\right] \times[0, \infty) \times[0, \infty)$. Note that $J(s)$ can be expressed as $J(s)=p(\xi(s))$, where $p(s, m, v):\left[0, t_{0}\right] \times[0, \infty) \times[0, \infty) \rightarrow \mathbb{R}$ is continuous, bounded, and measurable, with bounded left-hand derivatives with respect to $s$ and $v$. It is easily seen that

$$
A p(s, m, v)=\alpha_{1}(v)\left(\mathrm{E}\left[g_{1}\left(m+X_{1}\right)\right]-g_{1}(m)\right)-\left(\bar{y}_{2-}^{\prime}\left(t_{0}-s\right)+c_{1}^{\prime}(s)\right) \quad \text { for } s \leq t_{0} .
$$

The rest of the proof follows that of Theorem 4.

Summarizing, the solution to the double stopping problem is given by

$$
\mathrm{E}\left[Z\left(\tau_{1}^{*}, \tau_{2}^{*}\right)\right]=\mathrm{E}\left[J\left(\tau_{1}^{*}\right)\right]=\gamma\left(M_{0}, T_{1,0}\right)=\gamma(0,0),
$$

where $\tau_{1}^{*}$ and $\tau_{2}^{*}$ are defined according to Theorem 6 and Theorem 4 , respectively.

\section{Examples}

The form of the solution means that it is difficult to calculate analytically. In this section we present examples for which an analytic solution can be calculated.

Proposition 2. If the process $\zeta_{2}(t)=A p^{s, m}\left(\xi^{s}(t)\right)$ has decreasing paths then the second optimal stopping time is given by $\tau_{2, n}^{*}=\inf \left\{t \in\left[T_{2, n}, t_{0}\right]: A p^{s, m}\left(\xi^{s}(t)\right) \leq 0\right\}$. On the other hand, if $\zeta_{2}(t)$ has nondecreasing paths then the second optimal stopping time is equal to $t_{0}$. Similarly, if the process $\zeta_{1}(s)=A p(\xi(s))$ has decreasing paths then the first optimal stopping time is given by $\tau_{1, n}^{*}=\inf \left\{s \in\left[T_{1, n}, t_{0}\right]: A p(\xi(s)) \leq 0\right\}$. On the other hand, if $\zeta_{1}(s)$ has nondecreasing paths then the first optimal stopping time is equal to $t_{0}$.

Proof. We conclude from (14) that

$$
\mathrm{E}\left[Z\left(s, \tau_{2, n}^{*}\right) \mid \mathcal{F}_{s, n}\right]=Z\left(s, T_{2, n}\right)+\mathrm{E}\left[\int_{T_{2, n}}^{\tau_{2, n}^{*}}\left(A p^{s, m}\right)\left(\xi^{s}(z)\right) \mathrm{d} z\right] \text { a.s. }
$$

Applying the results of [6] completes the proof. 
Corollary 1. If $S_{2}$ has exponential distribution with constant hazard rate $\alpha_{2}, g_{2}$ is increasing and concave, $c_{2}$ is convex, $t_{2, n}=T_{2, n}$, and $m_{n}^{s}=M_{n}^{s}$, then

$$
\left.\tau_{2, n}^{*}=\inf \left\{t \in\left[t_{2, n}, t_{0}\right]: \alpha_{2}\left(\mathrm{E}\left[g_{2}\left(m_{n}^{s}+X_{2}-m\right)\right]-g_{2}\left(m_{n}^{s}-m\right)\right)\right] \leq c_{2}^{\prime}(t-s)\right\},
$$

where $s$ is the time when the angler changes fishing location. Moreover, if $S_{1}$ has exponential distribution with constant hazard rate $\alpha_{1}, g_{1}$ is increasing and concave, $c_{1}$ is convex, $t_{1, n}=T_{1, n}$, and $m_{n}=M_{n}$, then

$$
\tau_{1, n}^{*}=\inf \left\{s \in\left[t_{1, n}, t_{0}\right]: \alpha_{1}\left(\mathrm{E}\left[g_{1}\left(m_{n}+X_{1}\right)\right]-g_{1}\left(m_{n}\right)\right) \leq c_{1}^{\prime}(s)\right\} .
$$

Proof. The forms of $\tau_{1, n}^{*}$ and $\tau_{2, n}^{*}$ are given in Proposition 2. Let us observe that, by our assumptions, $\zeta_{2}(t)=\alpha_{2} \Delta_{2}\left(M_{t}^{s}-m\right)-c_{2}^{\prime}(t-s)$ has decreasing paths for $t \in\left[T_{2, n}, T_{2, n+1}\right)$. It suffices to prove that

$$
\zeta_{2}\left(T_{2, n}\right)-\zeta_{2}\left(T_{2, n}^{-}\right)=\alpha_{2}\left(\Delta_{2}\left(M_{n}^{s}-m\right)-\Delta_{2}\left(M_{n-1}^{s}-m\right)\right)<0 \quad \text { for all } n \in \mathbb{N} .
$$

It remains to check that $\bar{y}_{2-}^{\prime}\left(t_{0}-s\right)=0$. We see that $\tau_{2}^{*}=\tau_{2}^{*}(s)$ is deterministic, which is clear from (19). Let us note that if $s \leq t_{0}$ then combining (14), (15), and (17) gives

$$
\gamma^{s, m}(m, s)=\mathrm{E}\left[Z\left(s, \tau_{2}^{*}\right) \mid \mathcal{F}_{s}\right]=Z(s, s)+\mathrm{E}\left[\int_{s}^{\tau_{2}^{*}}\left(A p^{s, m}\right)\left(\xi^{s}(z)\right) \mathrm{d} z \mid \mathcal{F}_{s}\right] .
$$

By Remark 3, it follows that

$$
\bar{y}_{2}\left(t_{0}-s\right)=\mathrm{E}\left[\int_{s}^{\tau_{2}^{*}(s)}\left(A p^{s, m}\right)\left(\xi^{s}(z)\right) \mathrm{d} z\right]=\int_{s}^{\tau_{2}^{*}(s)}\left(\alpha_{2} \Delta_{2}(0)-c_{2}^{\prime}(z-s)\right) \mathrm{d} z,
$$

and this yields

$$
\begin{aligned}
\bar{y}_{2-}^{\prime}\left(t_{0}-s\right) & =\int_{s}^{\tau_{2}^{*}(s)} c_{2}^{\prime \prime}(z-s) \mathrm{d} z+\tau_{2}^{* \prime}(s)\left(\alpha_{2} \Delta_{2}(0)-c_{2}^{\prime}\left(\tau_{2}^{*}(s)-s\right)\right)-\left(\alpha_{2} \Delta_{2}(0)-c_{2}^{\prime}(0)\right) \\
& =c_{2}^{\prime}\left(\tau_{2}^{*}(s)-s\right)-c_{2}^{\prime}(0)-\left(\alpha_{2} \Delta_{2}(0)-c_{2}^{\prime}(0)\right) \\
& =0 .
\end{aligned}
$$

The second part of the proof follows similarly to the first part.

Corollary 2. If, for $i=1,2$, the functions $g_{i}$ are increasing and convex, the functions $c_{i}$ are concave, and the $S_{i}$ have exponential distribution with constant hazard rate $\alpha_{i}$, then $\tau_{1, n}^{*}=$ $\tau_{2, n}^{*}=t_{0}$ for $n \in \mathbb{N}$.

Proof. The proof is a straightforward consequence of Proposition 2. It suffices to check that $\bar{y}_{2-}^{\prime}\left(t_{0}-s\right)$ is nonincreasing with respect to $s$. First observe that $\tau_{2}^{*}(s)=t_{0}$. By (20), it is obvious that $\bar{y}_{2-}^{\prime}\left(t_{0}-s\right)=\alpha_{2} \Delta_{2}(0)-c_{2}^{\prime}\left(t_{0}-s\right)$. This completes the proof.

\section{Conclusions}

In this paper we presented the solution to the double stopping problem in the fishing model for a finite horizon. The analytical properties of the reward function in the single stopping problem played a crucial rule in our considerations and allowed us to extend the problem to double stopping. Let us note that, by repeating the considerations of Section 4, it is easy to generalize our model and the solution to the multiple stopping problem. Key assumptions in our model were connected with the properties of distribution functions. Hypothetical extensions of the above model include admitting general distributions and an infinite horizon. 


\section{Acknowledgement}

I would like to thank my supervisor Professor Krzysztof Szajowski for valuable suggestions that helped me to write this paper.

\section{References}

[1] Boshuizen, F. A. And Gouweleeuw, J. M. (1993). General optimal stopping theorems for semi-Markov processes. Adv. Appl. Prob. 25, 825-846.

[2] Brémaud, P. (1981). Point Processes and Queues. Springer, New York.

[3] Davis, M. H. A. (1993). Markov Models and Optimization (Monogr. Statist. Appl. Prob. 49). Chapman and Hall, New York.

[4] Ferenstein, E. And Sierociński, A. (1997). Optimal stopping of a risk process. Applicationes Math. 24, 335-342.

[5] Ferguson, T. S. (1997). A Poisson fishing model. In Festschrift for Lucien Le Cam, eds D. Pollard et al., Springer, New York, pp. 235-244.

[6] JENSEN, U. AND Hsu, G. H. (1993). Optimal stopping by means of point process observations with applications in reliability. Math. Operat. Res. 18, 645-657.

[7] Karpowicz, A. ANd SzaJowsKi, K. (2007). Double optimal stopping of a risk process. Stochastics 79, 155-167.

[8] Kramer, M. and Starr, N. (1990). Optimal stopping in a size dependent search. Sequent. Anal. 9, 59-80.

[9] Rolski, T., Schmidli, H., Schimdt, V. ANd Teugels, J. (1998). Stochastic Processes for Insurance and Finance. John Wiley, Chichester.

[10] Starr, N. (1974). Optimal and adaptive stopping based on capture times. J. Appl. Prob. 11, $294-301$.

[11] Starr, N. And Woodroofe, M. (1974). Gone fishin': optimal stopping based on catch times. Tech. Rep. 33, Department of Statistics, University of Michigan.

[12] Starr, N., Wardrop, R. AND Woodroofe, M. (1976). Estimating a mean from delayed observations. Z. Wahrscheinlichkeitsth 35, 103-113. 